\title{
Maria Corti o dell'entusiasmo
}

\author{
Luca Carlo Rossi
}

I ritratti degli studiosi somigliano spesso a floreali vite di santi (vergate da devoti cultori) o a polverose fotografie in pose rigide (quando non sono epigrafi tombali): quello di Maria Corti, proposto nel Dialogo in pubblico (Milano: Rizzoli, 1995), non rientra in nessuna delle tipologie individuate, ma è una ripresa dal vivo, vivace e mobile come l'originale, effettuata da Cristina Nesi con la formula dell'intervista (intelligente, a differenza di quanto ormai capita quasi sempre) effettuata fra il luglio 1992 e quello 1994. L'immagine che ne esce equivale a una di quelle istantanee che colgono i tratti caratteristici di una persona sorpresa in un gesto tutto suo, in piena naturalezza, e li bloccano vivi in una eternità d'istante. Del resto non poteva essere altrimenti nel caso di Maria Corti, costituzionalmente estranea alle rigidità accademiche e acuta sperimentatrice di molte novità nel campo della comunicazione. E subito, ad apertura di libro, la conferma della sua apertura mentale viene dalla riflessione sui vantaggi dell'intervista, definita uno "spazio sperimentale": l'imprevedibilità delle domande e delle risposte per $\mathrm{i}$ due interlocutori e la chiarificazione che l'intervistato fa anche a se stesso di fatti della sua vita. È certo anche per questi motivi che altri eminenti personaggi della cultura, di fama consolidata e spregiudicati come la Corti, hanno scelto di stendere un resoconto pubblico della loro attività pluriennale attraverso l'intervista, che in realtà sono poi essi a condurre con accorta civetteria e discrezione: penso a Gianfranco Contini nella conversazione con Ludovica Ripa di Meana (Diligenza e voluttd), a Ernst H. Gombrich in colloquio con Didier Eribon (Il linguaggio delle immagini) e ancora a Carlo Dionisotti nell'intervista televisiva Verba manent a cura di Renzo e Claudia Villa.

Chi ha la ventura di conoscere personalmente Maria Corti la troverà qui appassionata e vulcanica come sempre; chi invece ne conosce gli scritti ritroverà il piglio, la verve singolare, l'intelligenza inesauribile che l'hanno subito resa un punto di riferimento, una istituzione del mondo culturale milanese, pavese, italiano e internazionale.

Pervade queste pagine un senso di concretezza, uno slancio vitale che si riconosce come profondo senso morale, senza che mai lo si definisca tale: il dovere, che è insieme un piacere, di capire il mondo in tutte le sue mani- 
festazioni: "Non si dovrebbe mai vivere fuori del proprio tempo. Per deludente che esso sia, e il posto dove siamo per guardare sia indietro che avanti, per percorrere la nostra strada [...] per conoscere la forma del nostro destino" (154). E ancora, a chiusura del ricordo dei seminari svizzeri di Bigorio, organizzati da padre Pozzi, quando, dentro le mura di un antico convento, senza divisioni gerarchiche, si discuteva liberamente attorno alla nascente edizione delle opere di Marino, e la sera "ci si riuniva a bere e a chiacchierare nella cantina del convento, dove c'erano vecchi tavoli odorosi di legno, castagno o noce": "Là c'era qualcosa di più di un incontro di studiosi, una passione intellettuale nell'aria, qualcosa con una nuova identità umana che si era creata da sé in tutti noi. Come dirlo... senza retorica? Meglio fermarsi qui. Basta aver salvato qualcosa dalle 'forbici del tempo' di montaliana memoria" (191). Così come altamente morale è il significato attribuito alla ricerca e allo studio che emerge dalla riflessione sulla cosiddetta morte della semiotica, alla cui diffusione la Corti ha dato un apporto decisivo: "Il passato degli anni Settanta [...] corre il rischio di essersi già consumato e non esserci più nella memoria storica generale, mentre vi sono studiosi che, senza alcun rumore pubblicitario, continuano ad approfondire problematiche, ad applicare quanto viene da proposte semiotiche, a indagarne gli effetti se applicate ai testi letterari. Sembra una lotta utopica, ma non lo è affatto. Questo lavoro silenzioso, corroborante non può dare nell'occhio nella nostra civiltà dello spettacolo, perché spettacolare non è, ma proprio per questo costruisce" (119).

Accanto alla operosità alacre di Maria Corti risalta la cordiale umanità, la assoluta disponibilità personale, doti rare che suscitano la simpatia dei giovani dai quali è circondata affettuosamente anche dopo la fine dei loro studi e che rendono per lei prezioso il sentimento dell'amicizia discreta ma salda. Un esercizio, questo, testimoniato da alcuni episodi, narrati con un estremo pudore per l'intimità privata. Ricordo i due, speculari, legati all'uomo, singolare al pari dell'intellettuale, Giorgio Manganelli "ipocondriaco e ilare, scontroso e generosissimo [...] capace di gesti imprevisti, ma memorabili" (201). "Direi che la sua vita fu di quelle in cui niente avviene senza complicazioni. Mettiamo il grande amore con Alda Merini. Il lato invisibile delle cose ha fatto sì che le loro profonde stranezze si siano manifestate a Milano con un'ammirevole coincidenza. Io vedendoli pensavo a questo, ma non credo che loro ci pensassero [...] I fatti parevano essere emanazioni del destino: loro due si amavano intensamente, sfuggivano a tutte le le resistenze familiari (lui aveva moglie e una bambina bionda, lei aveva sedici anni), vi sfuggivano in un albergo di via San Gottardo. Oggi a guardarlo quel luogo e squallido, albergo di bassa categoria, ma in quel luogo tetro c'è stato, nascosto in una stanza, lo splendore di una grande passione di due artisti [...] solo Dio poteva sapere che fine avrebbe fatto quel loro amore. Adesso, anno 1994, lei è cresciuta molto come poetessa [...]. Lui è morto da pochi anni" (202). "Era 
l'estate del 1970 ed ero a Pellio Intelvi nella mia vecchia casa solitaria. In seguito ad alcune vicende del tutto personali, ebbi la sensazione che niente sarebbe stato come prima nella mia esistenza [...] La prima cosa che feci fu quella di telefonare a Roma a Manganelli. Gli dissi tout court che non mi andava più di stare al mondo. 'Sei al mondo dove?' chiese. 'A Pellio'. 'Scendi subito a Milano, prendi domattina un aereo. Io sarò all'aeroporto'. Nel 1970 Manganelli guidava l'automobile, malissimo. Ricordo che mentre mi portava a casa sua ebbi più volte la paura di morire in uno scontro. Me ne stupii perché il giorno prima avevo invece la paura opposta, quella di stare al mondo. Giorgio ed Ebe Flamini furono meravigliosi, mi trattavano come una convalescente, lui con musica di Vivaldi e inni medievali russi, lei con selvaggina speciale della zona dei Castelli. In quella settimana sperimentai cos'è un'amicizia autentica di fronte alle amicizie letterarie di cartapesta che tanto abbondano fra noi, soprattutto via telefono" (204).

Il libro possiede la stessa mobilità dell'intelligenza di Maria Corti e si configura come un viaggio attraverso la cultura in compagnia di una guida esperta ma sempre disposta a vedere cose nuove nel paesaggio che non trova mai monotono: il discorso si illumina di subite accensioni e si aprono piste inattese, improvvise prospettive. È dunque difficile darne un resoconto senza riproporlo integralmente; ho deciso così di selezionare qualche pagina dove più traspare l'entusiasmo della Corti -la sua cifra più caratteristica-, ciascuna collegata ai suoi principali campi di azione, con la speranza di stuzzicare la voglia di una lettura integrale, prima del Dialogo in pubblico, e poi di almeno uno dei tanti e diversi testi della studiosa e scrittrice, il cui elenco, steso da R. Saccani e integrato da C. Nesi (211-83), riserva non poche sorprese e documenta insieme l'assoluta versatilità e la prolificità della Corti (720 voci fra volumi, studi in riviste, recensioni, articoli su quotidiani dal 1937 al 1994). ${ }^{1}$ Sicché non sarà improprio estenderle l'epiteto omerico da lei riservato al banchiere mecenate Raffaele Mattioli, di polytropos (137): un'associazione che chiama in causa il primo destinatario della qualifica, Ulisse, un personaggio spesso ricorrente nell'opera della Corti certo per affinità dovuta alla comune curiositas intellettuale, che, se fu dannosa nel modello, si è invece sempre risolta per lei in fonte di piacere e di felicità mentale, per riprendere il titolo di uno dei suoi volumi dedicati a Dante.

Maria Corti studiosa: la storica della lingua

Storia della lingua italiana è la disciplina di cui Maria Corti è stata titolare all'Università dal 1962, prima a Lecce poi a Pavia: approdatavi dopo ventitré anni di "tunnel", costituito dalla compresenza delle attività di insegnamento (prima a Chiari, presso Brescia, poi a Como, infine al classico Beccaria di 
Milano), di ricerca e di invenzione, la Corti ha esteso le sue indagini sull'intero arco cronologico della materia. Ai due estremi si collocano le inchieste sotto ricordate: nate da un interesse puntuale si allargano a macchia comprendendo situazioni e persone impreviste, a testimonianza di come l'intera realtà sia per la Corti un inesauribile accumulatore di significati.

Lo studio della lingua di un lapidario veneto, in un codice estense del Trecento, porta la studiosa a Treviso, alla ricerca di conferme per la localizzazione linguistica negli Archivi di Stato e nei documenti della Biblioteca Capitolare. "Vi ho trovato due preti bizzarri, il direttore della Capitolare che aveva paura a lasciar vedere le pergamene e uno stranissimo monsignore che aveva creato una teoria dell'inesistenza dei poeti provenzali [...]. Gianfranco Contini mi aveva dato una lettera di presentazione per quel mirabile intellettuale che era il leopardista Gino Scarpa e una per Ciro Cristofoletti, direttore allora della libreria più importante di Treviso, la libreria Canova. Con il salvacondotto continiano passai straordinarie serate trevisane sulle panchette pubbliche in periodo quasi estivo o nelle trattorie a discorrere con gli intellettuali locali di letteratura o ad ascoltare storie con Comisso a protagonista. $\mathrm{Ci}$ tornai in settembre. E certo allora non immaginavo che nei tardi anni Settanta e poi negli anni Ottanta sarei stata invitata a entrare nelle giurie del primo e del secondo premio Treviso Comisso [...]. Ricordo che la prima giuria del premio lo aveva assegnato a Giorgio Manganelli. Egli fece un discorso strepitoso che lascid incantata e un po' spaventata la gioiosa borghesia della citta. Ma ricordo anche la visita notturna che volle fare con me alla vecchia Treviso, alle strade pochissimo illuminate e interamente a porticato, al vicolo dei Buranelli, al corso dei due fiumi. [...]. Lo vedo ancora avanzare nella penombra della vecchia Treviso notturna, vestito di nero, con qualcosa di fantomatico nella figura e quasi lugubre" (66-69).

Nel 1976, epoca di proteste, nasceva il cosiddetto Movimento, costituito da vari gruppi giovanili di vasta provenienza, universitari piuttosto sofisticati e il nuovo proletariato, simpatizzanti di Autonomia operaia, "di cui avevano rifiutati gli aspetti militar-politici, le simpatie nei confronti di un nascente terrorismo, a favore invece di aspetti spontaneistico-creativi" (143). Come storica della lingua attenta al linguaggio studentesco, ripreso anche nel romanzo Il ballo dei sapienti del 1966, la Corti si interessa al "vitalismo estetico" del collettivo che si esprimeva attraverso Radio Alice, fondata a Bologna sempre nel 1976, e ricercava una nuova via di espressione linguistica riagganciandosi in via analogica all'avanguardia futurista, "che pure usava un linguaggio colto e un po' sofisticato come il nostro Collettivo", ma restando in contatto con la fascia bassa della società. "Con Radio Alice torna sul piano della lingua la proposta avanguardistica e neoavanguardistica di far saltare la 'dittatura del significato', di operare una scrittura trasversale 'maodadaista', di porre piccole bombe a orologeria per distruggere le strutture di una razionalità linguistica di natura borghese, dopo di che si contribuirebbe 
alla caduta della dittatura del politico. Mitologie d'antan! postillerei con Giovanni Palmieri, studioso del fenomeno" (144-45).

Non poteva infine mancare nello spettro delle esplorazioni linguistiche della Corti il rock italiano di fine anni Settanta. "Mentre mi trovavo con alcuni ricercatori e giovani colleghi nel teatro [di Piacenza], dove sul palcoscenico [Nanni] Balestrini recitava [le sue] Ballate della signorina Richmond [sul fervore rivoluzionario dei movimenti giovanili] con un accompagnamento di chitarra [...] cominciarono a piovermi sulla testa foglie di lattuga dal loggione [...] mentre sul palcoscenico arrivavano di sbieco anche carote, meno gradevoli a riceversi in testa. Seppi poi che l'iniziativa, vagamente goliardica, era stata degli Skiantos. Fu quella l'occasione in cui venni informata dai giovani pavesi, che la sapevano lunga, trattarsi dei primi gruppi bolognesi del rock italiano. In seguito un mio allievo, Massimo Depaoli, chiese di laurearsi sulla lingua delle canzoni dei gruppi rock italiani. Acconsentii a seguirlo e ne nacque un bel volume edito dall'editore Longo di Ravenna [...] che ormai a distanza di anni assume un valore storico per lo studio della canzone italiana [...]. Anche qui c'è un leader, che è Roberto Antoni, in arte Freak Antoni, intellettuale, musicista, scrittore, uno dei più creativi, inventore per esempio del rock 'demenziale', prodotto assai ironico quando non fornito di vera satira” (146-47).

Maria Corti studiosa: la filologa tra Medioevo e contemporaneità

L'interesse della Corti per il Medioevo è una costante che ha origine dalla sua tesi di laurea sulla latinità merovingia in testi agiografici minori, sotto la guida dell'amatissimo maestro Benvenuto Terracini. Poco incline a legarsi stabilmente a quell'ambito e alla prospettiva strettamente linguistica, la studiosa ha poi definito i periodi storici cui avrebbe maggiormente rivolto il suo interesse: il Duecento, il Quattrocento e l'epoca contemporanea. "Devo postillare che del Medioevo [...] hanno incominciato a interessarmi le grandi voci filosofiche oltre a quelle letterarie. Credo che proprio in questi anni si sia verificata in me la fusione vera fra la cultura che era stata rappresentata da Terracini e quella scoperta con Banfi [il filosofo con il quale la Corti prese la seconda laurea con una tesi su Africano Spir]. La mia ricerca sulle fonti del Fiore di virtu del 1959 ne è un chiarissimo indizio; e non e un caso che la tematica di questo saggio sia poi andata a finire nel 1983 nel mio volume più integralmente legato al pensiero filosofico medievale: La felicitd mentale" (74).

Ricordo la trilogia dantesca di Maria Corti (Dante a un nuovo crocevia, 1981; La felicitd mentale, 1983; Percorsi dell' invenzione, 1993) portatrice di autentiche novità, non da tutti pacificamente accettate. Il primo volume offre una riconsiderazione del De vulgari eloquentia alla luce della grammatica 
speculativa dei modisti o cultori della logica simbolica, nata a Parigi e insegnata a Bologna, con una rilettura della presenza dell'aristotelismo radicale o averroismo nella Commedia: "dal rifiuto netto nel canto dell'eretico Cavalcante a un'affascinante figurazione simbolica in quello di Ulisse, a una motivata rivalutazione dell'ultimo Sigieri [di Brabante] in Paradiso X" (Dante a un nuovo, 97). Nel secondo libro compaiono novità sulle fonti filosofiche e sui commenti ad Aristotele circolanti nella cultura duecentesca con una precisa lettura della grande canzone cavalcantiana Donna me prega, corredata delle fonti documentarie e, soprattutto, viene avanzata la proposta che il quarto dei trattati in cui si articola Il Convivio, vistosamente distante sul piano filosofico e linguistico dai primi tre, sia stato composto dopo un intervallo nel quale Dante modificò sensibilmente la propria impostazione ideologica e stese il De vulgari eloquentia. Per illustrare l'ultimo anello della catena, dedicato alle riflessioni sull'invenzione artistica, sulla genesi misteriosa dell'opera letteraria, sull'ineffabilità del discorso poetico, basta riportare uno stralcio dalla Premessa: “' 'A cosa del mondo assomiglia la musica?' si domandava Leibniz. La domanda tollera di essere estesa: 'A cosa del mondo assomiglia il linguaggio poetico?'. L'interrogativo in fondo ce lo poniamo oggi come se lo posero Dante e gli stilnovisti, sicché è lecito ripetere con Croce che ogni storia è storia contemporanea o con Lotman che il passato non finisce mai" (Percorsi dell' invenzione, VII).

Scavalco le ricerche filologiche su Sannazaro (75-76, 100-101), sul poemetto Delfilo assegnato a Francesco Colonna anche in seguito a spedizioni automobilistiche per la valle del Trebbia (81-82), sugli scritti del giovanissimo Leopardi (125-26), per arrivare al caso Fenoglio (206-208): "Certe volte la vita imbastisce con niente una lunga storia. Voglio dire che forse non $\mathrm{mi}$ sarei mai occupata in vita mia di Fenoglio se nell'estate del 1968, a Courmayeur in villeggiatura, tra una gita alpinistica e l'altra, non avessi preso in mano l'edizione einaudiana del Partigiano Johnny [nata dalla combinazione delle due redazioni del romanzo]. Leggere quel libro epico era un piacere straordinario, ma mi stupiva profondamente il fatto che ogni tanto provassi la sensazione di aver già letto un dialogo, un episodio, qualche splendida similitudine. Ricordo che ne parlai con Cesare Segre, compagno di gite a Courmayeur, gli dissi: 'Come mai succede questo se è l'ultima opera dello scrittore, edita postuma?'. Lui mi rispose con la consueta calma: 'L'essere edita postuma non vuol dire che sia l'ultima opera'. Tutto il futuro lavoro mio e dell'équipe che lavoro con me all'edizione einaudiana in tre volumi del 1978 sono nati da questo mio stupore del 1968". Secondo la Corti e i suoi collaboratori il Partigiano Johnny appartiene alla prima fase della produzione di Fenoglio e gli sarebbe servito in seguito come 'magazzino' dal quale trarre materiali per altre opere. "È accaduto che noti studiosi, che andavano per le vie del mondo, si imbatterono nel grande scrittore morto da poco e attratti si intrufolarono 
nell'universo delle Langhe. Così, per una disinvoltura attribuibile al Caso, nacque la questione Fenoglio. Dopo di che la critica ha creato attorno allo scrittore piemontese un caos labirintico al posto del personale rapporto di comunicazione con il lettore privilegiato da Fenoglio" (207-208).

A Maria Corti piace (usa proprio questo verbo) l'indagine poliziesca sul testo, ha il gusto per le ricerche indiziarie: perfetto risulta dunque l'epiteto di 'Perry Mason della letteratura' assegnatole ("con mio grande piacere", ammette) da un altro primario filologo, Giorgio Petrocchi (81). Dal suo entusiasmo sono scaturiti, accanto ad alcune eccellenti edizioni (fra le altre, quelle di Pietro Jacopo De Jennaro, Vita di san Petronio, Elio Vittorini, Ennio Flaiano, Gesualdo Bufalino), non pochi recuperi di quei fantasmi che abitano la letteratura dietro l'ombra dei grandi classici, gli anonimi e gli animatori culturali. Per tale aspetto la studiosa si dichiara ideale discepola di un "trio geniale" (98): Augusto Campana, Giuseppe Billanovich e Carlo Dionisotti, i quali "hanno dimostrato un'acribia filologica, storica e critica senza la quale sarebbero ancora molte le lacune nella conoscenza della nostra letteratura e della nostra storia" (99). In particolare la Corti elogia la Biblioteca dell'Università Cattolica di Milano $(99-100,165)$ alla cui ricchezza ed efficiente organizzazione deve molte occasioni di scoperta ("Lì ho passato proprio ore intellettualmente felici", 165), e segnala le doti di Billanovich che l'ha organizzata sul modello della Biblioteca Vaticana e della Biblioteca dell'Università di Friburgo. Di Billanovich ammira soprattutto "la tenace fiducia storica: [...] più la realtà è puntiforme, più lui arriva a descrivertela, magari a dirti con chi quel tal giorno si è incontrato il Petrarca, con chi ha fatto il viaggio da Padova a Roma, dove erano i manoscritti posseduti e postillati dal poeta e ora in giro per il mondo. Rara e preziosa una simile disponibilità costruttiva" (166).

$\mathrm{Va}$ infine ricordato che se anche alcune proposte ardite di Maria Corti non sono per tutti pienamente condivisibili, la passione, lo slancio e il brillio dell'intelligenza che le sostengono rendono sempre stimolante ogni sua nuova indagine; sintetizza efficacemente Contini recensendo La felicita mentale: "L'autrice è la prima a stupire e a gioire. Dickens fiutò subito che il nome di George Eliot celava una personalità femminile. Qui non v'è alcuno sforzo di dissimulazione. Da ciò anche la sua simpatica mancanza di cautela" (Contini, "Novità" 209).

Maria Corti promotrice di cultura: Il Fondo Manoscritti

Oltre che ad alcune attività culturali più tradizionali, quali la fondazione di riviste ("Alfabeta", "Strumenti critici", "Autografo") e la direzione di collane editoriali ("La Nuova Corona" di Bompiani), Maria Corti ha legato il proprio nome a una istituzione da lei inventata presso l'Università di Pavia: il "Fondo 
Manoscritti di autori moderni e contemporanei", che dal 1968 a oggi continua a raccogliere materiali preziosissimi, soprattutto grazie alla tenacia della sua ideatrice, non certo per il sostegno dello Stato italiano, che pose diverse difficoltà a dare l'autorizzazione per il dono che la Corti volle fargli, lasciando all'Università di Pavia alcuni manoscritti di Montale e di Bilenchi a lei regalati dagli autori e di Gadda dati da Roscioni. "A pensare quante fatiche costò creare questa istituzione dal titolo "Fondo Manoscritti di autori moderni e contemporanei", mi viene da dire a proposito dell 'intestazione con Salvador Dalì: 'Successione di parole che ha il merito di significare pressappoco niente' " (130-31).

Superato lo scoglio e nonostante la concorrenza di università americane, giapponesi e del collezionismo privato, il fondo ora copre uno spettro di due secoli: da testi dell'Ottocento (inizialmente non previsti) con epistole di Foscolo e dei suoi corrispondenti, oltre che di alcuni volumi in pelle del poeta "che tolgono il fiato per bellezza e rarita" (132), al materiale riguardante Emilio De Marchi, da una redazione inedita di Pianissimo di Sbarbaro (dono di Cesare Angelini, affettuosamente ricordato alle 62-65) a lettere della Banti, di Bilenchi, di Carlo Levi, agli epistolari diretti a Longhi, Terracini, al Fondo Saba, sottratto ad acquirenti americani con un prestito forzoso dai fondi universitari di ogni Facoltà dell'ateneo pavese; infine la segnalazione di una lettera di Montale diretta alla stessa Corti nel 1965 "che conto mantenere inedita finché sarò in vita" (138) sul problema se restare a Milano, dopo la morte della moglie, la celebre Mosca, o se tornare a Firenze. Descrivendo questa sua creatura, alla quale ha poi dedicato l'originale 'fantasia' Ombre dal Fondo (1997), la Corti ha modo di parlare della scrittura in era del computer: "Non credo che l'uso del computer sia molto generalizzato nella fase della prima stesura di un'opera. Credo piuttosto che lo scrittore prevalentemente usi il computer al posto della macchina da scrivere, cioè nella fase in cui prima usava la macchina da scrivere. Per quel che ne so, i più fra gli scrittori amano ancora affidare al foglio bianco e silenzioso le prime idee, $\mathrm{i}$ primi passi verso l'invenzione. Certo scomparirà la ricchezza delle varianti, in quanto in genere il computer è usato per cancellarle. Se quanto diciamo è vero, un giorno, magari fra un secolo, la gente pagherà il bilgietto d'ingresso e andrà nei musei a guardare i manoscritti come ora va alle mostre dei codici miniati del XIII e XIV secolo. Una guida allora spiegherà alla gente riunita nelle sale del museo che un tempo nell'uomo c'era un rapporto diretto fra la sua oralità e la sua scrittura e quest'ultima proteggeva sul bianco della carta l'individualità di chi aveva la penna in mano e con essa produceva una sua personale calligrafia, che lo distingueva dagli altri che usavano la penna. La grafia della scrittura faceva parte della comunicazione, tanto che c'erano dei grafologi che studiavano sulla scrittura a mano il carattere di una persona. E accadeva anche che un uomo cambiasse un po' grafia a seconda dei momenti psicologici o dell'età. Odore di passato, commenterà la guida, odore di 
accaduto. A me l'idea della fine della scrittura a mano fa venire un groppo in gola, ma forse siamo in pochi a provare questa sensazione" (134).

\section{Maria Corti scrittrice: "L'ora di tutti"}

In parallelo con l'attività critica Maria Corti svolge quella di scrittrice creativa: fu un inizio quasi per passatempo nel 1946, dettato dalla solitudine di Chiari dove insegnava "lontanissima dai vecchi amici e dal mio mondo di studi" (194). I due aspetti convivono alternativamente e traggono reciproco vantaggio: "Sono convinta che a chi si dà alla narrativa può diventare persino vantaggioso essere su un altro versante un critico, un teorico. Può essergli utile la riflessione che modellare secondo una struttura la materia incoerente e vertiginosa del nostro immaginario è arduo, ma necessario. D'altra parte l'essere utenti in proprio dei processi inventivi dello scrivere può favorire l'operazione critica e quella stilistica; un vero critico dovrebbe sempre essere nei suoi prodotti uno scrittore; lo ha già detto Gianfranco Contini e ce ne ha già dato l'esempio" (57).

I libri narrativi di Maria Corti sono stati pochi (L'ora di tutti 1962, Il ballo dei sapienti 1966, Voci dal Nord Est: taccuino americano 1986, Il canto delle sirene 1989, Cantare nel buio 1991) ma sempre hanno riscosso successo di critica e di pubblico perché sanno combinare la materia di cui sono fatti i sogni con la sottile attenzione al dato concreto, colto, attraverso un sorvegliato concerto stilistico. Offrono l'altra faccia della Corti, perfettamente speculare a quella critica: acuta, sensibile e insieme lucidamente controllata; forse anche per questo l'autrice non li definisce romanzi, salvo Cantare nel buio (84).

Il disagio di parlare dei propri testi creativi ("Me ne dà già il pubblicarli, ma doverne poi anche parlare..." 84) non impedisce alla scrittrice di soffermarsi su ciascuno. Qui ho selezionato alcune osservazioni riservate al libro più apprezzato, che è anche il più tradotto, ossia L'ora di tutti "una polifonia di cinque voci d'oltretomba", sintetizza l'intervistatrice (84), "che descrivono ciascuna la propria presenza a Otranto sino all'attimo della morte" avvenuta durante la presa della citta da parte dei Turchi nel 1480. Il titolo assai suggestivo, prelevato dalla prima parte di un racconto del seicentista Quevedo ( $L$ a hora de todos y la Fortuna con seso) ma completamente rinnovato, "allude a quell'ora della vita in cui ciascuno può dare prova di sé, ora che viene per tutti e che può anche coincidere con la morte" (88-89).

"Con la struttura scelta non solo diversi racconti sulla stessa realtà venivano a intrecciarsi, quindi diverse verità si offrivano per leggere lo stesso fatto, ma il tutto passava attraverso il filtro straniante della memoria. La tematica proviene da un'area di mia ricerca storica in quanto ho lavorato a lungo sulla cultura del regno di Napoli nel Quattrocento. È curioso come la nostra mente assimili suggestioni così diverse, contemporaneamente artisti- 
che e scientifiche" (84). Molti però sono i fili che la memoria intreccia nel libro: il fascino del Salento, frequentato assai spesso dalla Corti fin dalla fine degli anni '40 (c'è un bel ricordo delle riunioni dell'Accademia Salentina fondata da Oreste Macrì coi poeti Girolamo Comi e Michele Pierri a Lucugnano, 49-53), un universo arcaico e magico fatto di un mirabile incontro di idee sacre, pagane e cristiane (14) dove sopravvive il grico, l'antica lingua parlata nell'area otrantina (13-16), i frequenti soggiorni estivi a Otranto (86), le reminiscenze delle letture agiografiche (24), le poesie del Caracciolo e del Galeota che celebrano le gesta dei lontani pescatori otrantini, le cronache sulla guerra d'Otranto, specie quelle di Antonio De Ferrariis, detto il Galateo, e quella in ottica turca di Ibn Kemal ("dove i 'porci infedeli' erano i cristiani e i figli di Dio i turchi: due microcosmi simmetrici e speculari” 87), le novità librarie statunitensi di quegli anni come l'Antologia di Spoon River di Edgar Lee Master, Il ponte di San Luis Rey e la commedia Piccola citta di Thornton Wilder (85-87). "Direi però che la vera fonte del mio libro è stata Otranto con le sue memorie e leggende, i sogni di vita e la irripetibile meraviglia del morire, i nomi dei decapitati scritti su una lapide al colle della Minerva, le ossa dei poveri pescatori racchiusi nelle bacheche della cattedrale" (87).

Al termine di questa cursoria rassegna non pare improprio riportare un monito sul nostro tempo: "Nella nostra epoca due sono i grandi pericoli: la fretta e la nozione di novità. Diceva Roger Caillois: la novità in sé è passeggera, la perfezione resta. [...] La fretta e la novità tolgono alla letteratura e alle arti quel meraviglioso senso di vertigine che danno in montagna le cose viste da grandi altezze" (160-61). È un'ottima sintesi coerente con l'atteggiamento aperto alla vita caratteristico di Maria Corti, che non appare molto disturbata dal rumore di fondo della vita attuale, divisa com'è fra un modo di vivere raccolto, quasi da otium umanistico, e una fondamentale curiositas verso le sorprese della vita, come pure verso quello che lei chiama il potere delle cose inutili (208).

Chiudo con un omaggio alla intelligenza delle donne, che ha davvero qualcosa di particolare, anche se, come avverte Madame de Staël, "il cervello non ha sesso" (182), citando alcune parole di un'altra artista (qualifica da intendersi in senso etimologico e quindi valida anche per Maria Corti) diversissima per professione e temperamento, ma simile alla Corti per tenacia e intelligenza, per la compresenza di studio interpretativo e attuazione in proprio. È l'epilogo delle Lezioni di canto alla Julliard School of Music di New York tenute fra il 1971 e il 1972 da Maria Callas. Fatte le opportune differenze, credo che la lezione di metodo e di vita coincida con quella luminosamente attuata da Maria Corti.

[...] c'è una cosa che vi chiedo: che i nostri sforzi non siano sprecati, che non dimentichiate il poco che vi ho dato. Prendetelo e applicatelo ad altre parti, in modo che il vostro fraseggio, la vostra dizione, la vostra conoscenza, e il vostro coraggio ne 
escano accresciuti; specialmente il vostro coraggio. Non pensiate che il canto sia una carriera facile. E il lavoro di una vita; non finisce qui. Come miei futuri colleghi, dovete andare avanti. Lottate contro le cattive tradizioni; ricordate, siamo i servitori di persone migliori di noi: i compositori. Loro hanno creduto; noi dobbiamo credere. (309)

\section{Univeristd Cattolica del Sacro Cuore, Brescia}

\section{NOTA}

1 Chi volesse avere notizie dettagliate sull'attività di Maria Corti può consultare il punto 8 della Bibliografia annessa al Dialogo in pubblico, 270-83. Segnalo in particolare Stussi; e Villa.

\section{OPERE CITATE}

Callas, Maria. Lezioni di canto alla Julliard School of Music. Traduzione italiana di Luigi Spagnol. Milano: Longanesi, 1987.

Contini, Gianfranco. "Novità su Dante e Cavalcanti”. Corriere della Sera, 16 ottobre 1983; poi in Ultimi esercizî ed elzeviri (1986-1987). Torino: Einaudi, 1988. 205-9.

Diligenza e voluttà. Ludovica Ripa di Meana intervista Gianfranco Contini. Milano: Mondadori, 1989.

Corti, Maria. L'ora di tutti. Milano: Feltrinelli, 1962.

-. Il ballo dei sapienti. Milano: Mondadori, 1966.

Dante a un nuovo crocevia. Firenze: Le Lettere, 1981.

La felicità mentale. Torino: Einaudi, 1983.

Voci dal Nord Est: taccuino americano. Milano: Bompiani, 1986.

Il canto delle sirene. Milano: Bompiani,1989.

. Cantare nel buio. Milano: Bompiani, 1991.

Percorsi dell' invenzione. Torino: Einaudi, 1993.

Dialogo in pubblico. Intervista di Cristina Nesi. Milano: Rizzoli, 1995.

Ombre dal Fondo. Torino: Einaudi, 1997.

Dionisotti, Carlo. Verba manent. Video. Ed. Renzo e Claudia Villa. Milano: Bruno Mondadori, 1991.

Eribon, Didier, e Emst H. Gombrich.Il linguaggio delle immagini. Torino: Einaudi, 1994.

Stussi, Alfredo. "Storia della lingua con Maria Corti". Belfagor 45 (1990): 669-702.

Villa, Claudia. "Maria Corti”. Belfagor 47 (1992): 543-56. 